\title{
LACUNARY SETS FOR GROUPS AND HYPERGROUPS
}

\section{CATHERINE FINET}

(Received 28 June 1991)

Communicated by C. Sutherland

\begin{abstract}
In this paper, we generalize the classical F. and M. Riesz theorem to compact groups and compact commutative hypergroups. The group $S U(2)$ of unitary matrices is also studied.

1991 Mathematics subject classification (Amer. Math. Soc.): 22 C 05.
\end{abstract}

\section{Introduction}

F. and M. Riesz proved the following result ([21], p. 335).

(Classical) F. AND M. Riesz THeOREM. Let $\mu$ be a measure on the unit circle $\mathbb{T}=\left\{e^{i \theta}, 0<\theta \leq 2 \pi\right\}$ whose Fourier coefficients

$$
\hat{\mu}(n)=\int_{0}^{2 \pi} e^{-i n \theta} d \mu(\theta)
$$

with negative index are equals to zero. Then $\mu$ is absolutely continuous with respect to Lebesgue measure.

J. H. Shapiro gave a new proof of this theorem based on a study of duals of subspaces of $L^{p}(\mathbb{T})$ for $0<p<1$ [22]. His ideas were used by $G$. Godefroy for the study of Riesz subsets of commutative discrete groups [10]. In another direction R. G. M. Brummelhuis generalized Shapiro's methods to compact metrizable groups whose center contains a circle group [3].

(C) 1993 Australian Mathematical Society 0263-6115/93 \$A2.00+0.00 
In this paper, we give extensions of the classical F. and M. Riesz theorem to compact groups and compact commutative hypergroups. See [15, 23, 24] for the definition and the fundamental properties of hypergroups. A hypergroup is a locally compact space which has enough structure so that a convolution on the space of finite regular Borel measures can be defined. Classical examples are the space of conjugacy classes of a compact group, spaces of orbits in a locally compact group of automorphisms and double-cosets of certain non-normal closed subgroups of a compact group. The class of hypergroups includes the class of locally compact topological groups.

We briefly describe the contents of this paper. In Section 2 we recall some basic properties of hypergroups. In Section 3 we construct approximate units of the space $L^{1}(K)$ for $K$ a compact hypergroup. In Section 4, we study lacunary sets in the dual of a compact hypergroup. We first extend a result of R. E. Dressler and L. Pigno [7]: the union of a Riesz set and a Rosenthal set is a Riesz set. We also investigate the class of Riesz sets, nicely placed sets and Shapiro sets in the dual object of a compact commutative hypergroup. Shapiro sets are Riesz sets (Theorem 4.6), for which the Mooney-Havin theorem extends. Following $[10,17]$, we use the localization technique to construct nicely placed sets. The stability by union is studied and extensions to the Mooney-Havin theorem are given. Section 5 is devoted to some examples, we consider in particular the hypergroup $K$ of the conjugacy classes of the compact group $S U(2)$ and construct nicely placed sets in the dual $\widehat{K}$. In Section 6 , we use techniques of infinite dimensional Banach space theory to study $\Lambda(1)$-sets in the dual object of compact hypergroups and give non-commutative extensions of G. F. Bachelis and S. E. Ebenstein's result [1] and of F. Lust-Piquard's result [16]. We also answer a question of R. G. M. Brummelhuis [5]. In Section 7, we generalize a result of G. Godefroy [10] to compact groups whose center contains a copy of the circle group; we improve a result of $R$. G. M. Brummelhuis $[4,5]$. We also give some applications to the unit sphere of $\mathbb{C}^{n}(n \geq 2)$ and to the Bergman-Shilov boundary of a bounded symmetric domain.

NotATION. Let $K$ be a compact group or a compact hypergroup. We denote by $\mathscr{C}(K)$ the space of continuous functions on $K$ and by $\mathscr{M}(K)$ the dual of $\mathscr{C}(K)$, the space of finite Borel measures on $K$. We denote by $\mathscr{M}^{+}(K)$ the subset of positive measures in $\mathscr{M}(K)$ and $D(K)$ the algebra generated by the discrete measures. We denote by $\delta_{x}$ the Dirac measure at the point $x$. We denote by $\mu_{a}$ (resp. $\mu_{s}$ ) the absolute continuous (resp. singular) part of a measure $\mu$ in $\mathscr{M}(K)$ with respect to the Haar measure. The $L(1, \infty)$ ("weak $L^{1}$ ") quasi-norm is defined by

$$
\|f\|_{1, \infty}=\sup _{\lambda \geq 0}\{\lambda P(|f| \geq \lambda)\} \text {. }
$$


We denote by $B(X)$ the closed unit ball of a Banach space $X$ and by $I_{E}$ the characteristic function of the set $E$.

\section{Basic properties of hypergroups}

Our main reference for hypergroups is [15]. Let us recall the definition of a hypergroup.

Definition 2.1. Let $K$ be a locally compact Hausdorff space. The space $K$ is a hypergroup if there exists a binary mapping $(x, y) \rightarrow \delta_{x} \star \delta_{y}$ of $K \times K$ into $\mathscr{K}^{+}(K)$ satisfying the following conditions.

(1) The mapping $\left(\delta_{x} \star \delta_{y}\right) \rightarrow \delta_{x} \star \delta_{y}$ extends to a bilinear associative operator $\star$ from $\mathscr{M}(K) \times \mathscr{M}(K)$ into $\mathscr{M}(K)$ such that

$$
\int_{K} f d(\mu \star \nu)=\int_{K} \int_{K} \int_{K} f d\left(\delta_{x} \star \delta_{y}\right) d \mu(x) d \nu(y)
$$

for all continuous functions $f$ on $K$ vanishing at infinity.

(2) For each $x, y \in K$, the measure $\delta_{e} \star \delta_{y}$ is a probability measure with compact support.

(3) The mapping $(\mu, \nu) \rightarrow \mu \star \nu$ is continuous from $\mathscr{M}^{+}(K) \times \mathscr{M}^{+}(K)$ into $\mathscr{M}^{+}(K)$; the topology on $\mathscr{K}^{+}(K)$ being the cone topology.

(4) There exists $e \in K$ such that $\delta_{e} \star \delta_{y}=\delta_{x}=\delta_{x} \star \delta_{e}$ for all $x \in K$.

(5) There exists a homeomorphism involution $x \rightarrow x^{-}$of $K$ onto $K$ such that, for all $x, y \in K$, we have

$$
\begin{gathered}
\left(\delta_{x} \star \delta_{y}\right)^{-}=\delta_{y^{-}} \star \delta_{x^{-}}, \text {where } \delta_{x}^{-} \text {is defined by } \\
\int_{K} f(k) d \delta_{x}^{-}(k)=\int_{K} f\left(k^{-}\right) d \delta_{x}(k),
\end{gathered}
$$

and also,

$$
e \in \operatorname{supp}\left(\delta_{x} \star \delta_{y}\right) \text { if and only if } y=x^{-}
$$

where $\operatorname{supp}\left(\delta_{x} \star \delta_{y}\right)$ is the support of the measure $\delta_{x} \star \delta_{y}$.

(6) The mapping $(x, y) \rightarrow \operatorname{supp}\left(\delta_{x} \star \delta_{y}\right)$ is continuous from $K \times K$ into the space $\mathscr{C}(K)$ of compact subsets of $K$, where $\mathscr{C}(K)$ is given the topology whose subbasis is given by all

$$
\mathscr{C}_{U, V}=\{A \in \mathscr{C}(K): A \cap U \neq \varnothing \text { and } A \subset V\}
$$

where $U, V$ are open subsets of $K$.

Note that in general $\delta_{x} \star \delta_{y}$ is not necessarily a discrete measure. A hypergroup $K$ is commutative if $\delta_{x} \star \delta_{y}=\delta_{y} \star \delta_{x}$ for all $x, y$ in $K$. Let us first 
recall some properties of compact commutative hypergroups. Such a hypergroup $K$ carries a Haar measure $m$ such that $m(K)=1$ and $\delta_{x} \star m=m$ for all $x$ in $K$. If $f$ is a Borel function on $K$ and $x, y \in K$ then $x \star y$ is defined by $f(x \star y)=\int_{K} f d\left(\delta_{x} \star \delta_{y}\right)$. A complex-valued function $\chi$ on $K$ is said to be multiplicative if $\chi(x \star y)=\chi(x) \chi(y)$ for all $x$ and $y$ in $K$. The dual $\widehat{K}$ of $K$ is the space of characters; that is the space of multiplicative continuous functions $\chi$ on $K$ such that $\chi\left(x^{-}\right)=\overline{\chi(x)}$ for all $x$ in $K$. The space $\widehat{K}$ is an orthogonal basis for $L^{2}(K)$. If $K$ is compact then $\widehat{K}$ is discrete. Let us note that $\widehat{K}$ is not necessarily a hypergroup. For $\mu \in \mathscr{M}(K)$, the Fourier-Stieltjes transform $\hat{\mu}$ of $\mu$ is defined on $\widehat{K}$ by

$$
\hat{\mu}(\chi)=\int_{K} \bar{\chi} d \mu \text {, for all } \chi \in \widehat{K} .
$$

The mapping $\mu \rightarrow \hat{\mu}$ is a norm-decreasing $\star$-algebra isomorphism from $\mathscr{K}(K)$ into the space of bounded functions on $\widehat{K}$. We are also concerned with compact, not necessarily commutative, hypergroups $K$. Such hypergroups also carry a Haar measure and are unimodular. The dual object $\Sigma$ is then the set of equivalence classes of continuous irreducible representations of $K$. If $K$ is commutative, then $\Sigma$ is to be identified with $\widehat{K}$, the space of characters. In the non-commutative case complications arise because not all continuous irreducible representations of $K$ have representation space of dimension 1. However all the representation spaces have finite dimension when $K$ is compact [24]. Let us recall a few definitions and properties [24]. The Fourier-Stieltjes transform of a measure $\mu$ in $\mathscr{M}(K)$ is then defined by

$$
\hat{\mu}(\tau)=\int_{K} \bar{\tau} d \mu, \text { for each } \tau \in \Sigma
$$

It is an operator-valued function on $\Sigma$. The spectrum of a measure $\mu$ in $\mathscr{M}(K)$ is defined by

$$
\operatorname{spec} \mu=\{\alpha \in \Sigma, \hat{\mu}(\alpha) \neq 0\}=\operatorname{supp} \hat{\mu} .
$$

For any subset $\Lambda$ of $\Sigma$, we let

$$
\mathscr{M}_{\Lambda}(K)=\{\mu \in \mathscr{M}(K), \operatorname{spec} \mu \subset \Lambda\} .
$$

Let $\mathscr{T}(K)$ denote the space of trigonometric polynomials on $K: \mathscr{T}(K)=$ $\left\{f \in L^{1}(K): \operatorname{spec} f\right.$ is a finite set $\}$. If $\Lambda \subset \Sigma$, let $\mathscr{T}_{\Lambda}(K)=\{f \in \mathscr{T}(K)$ : $\operatorname{spec} f \subset \Lambda\}$.

\section{Approximate units in $L^{1}(K)$}

In this section, we construct approximate units in $L^{1}(K)$ for $K$ a compact hypergroup. We get a generalization of a result of J. Boclé [2, Theorem II, 
page 17], see also [22, Lemma 1.1]. In the following $K$ will denote a compact not necessarily commutative hypergroup, $m$ its Haar measure. We assume $m(K)=1$. The main result of this section is the following

THEOREM 3.1. Let $K$ be a compact hypergroup. There exists a net of functions $\left\{h_{\alpha}\right\}$ in $L^{1}(K)$ such that for all $\alpha$ :

(1) $h_{\alpha} \in \mathscr{T}(K)$.

(2) $\left\|h_{\alpha}\right\|_{1}$ is bounded.

(3) If $\mu \in \mathscr{M}_{s}(K)$ then the net $\left\{\mu \star h_{\alpha}\right\}$ converges in Haar measure to zero.

(4) If $f \in L^{1}(K)$ then the net $\left\{f \star h_{\alpha}\right\}$ converges in $L^{1}$-norm to $f$.

Proof. Consider $\mathscr{U}$ a basis of neighborhoods at $e$ consisting of symmetric sets. We direct the net in the usual way: $U \geq V$ if $U \subset V$. For $V \in \mathscr{U}$, let $f_{V}=m(V)^{-1} I_{V}$. We now prove that the net $\left\{\mu \star f_{V}\right\}_{V \in \mathscr{U}}$ converges in Haar measure to 0 when $\mu$ belongs to $\mathscr{M}_{s}(K)$. Since $\left|\mu \star f_{V}\right| \leq|\mu| \star f_{V}[15$, 6.1.B] we may without loss of generality suppose $\mu$ to be a positive measure. Let $\varepsilon>0$ and $a>0$. Since $\mu$ is a regular and singular measure on $K$ there exist a compact set $H$ and an open set $U$ such that $H \subset U \subset K$ and $\mu(U)=\mu(K)=\|\mu\|, \mu(U \backslash H)<\varepsilon a / 2, m(U)<\varepsilon / 2$. Define $\lambda$ in $\mathscr{M}(K)$ as follows:

$$
\lambda(B)=\mu(B \cap H) \text { for } B \text { a Borel subset of } K .
$$

Then $\mu=\lambda+\theta$ where $\theta(K)<\varepsilon a / 2$. By [15, 3.2.D], there is a neighborhood $W$ in $\mathscr{U}$ such that $W \star H \subseteq U$. By definition, one has:

$$
\left(\lambda \star I_{W}\right)(t)=\int_{K} I_{W}\left(y^{-} \star t\right) d \lambda(y)=\int_{K}\left(I_{W} \star \delta_{t}\right)(y) d \lambda(y)
$$

and,

$$
\left(\lambda \star I_{W}\right)(t)=\int_{(W \star t) \cap H}\left(I_{W} \star \delta_{\ell}\right)(y) d \mu(y) .
$$

By $[15,4.1 . \mathrm{B}]$, the set $(W \star t) \cap H$ is empty if and only if the set $\{t\} \cap\left(W^{-} \star H\right)$ is empty; that is $t$ does not belong to the set $W^{-} \star H$ and by the symmetry of $W, t$ does not belong to $W \star H$. It follows that if $t$ does not belong to $U$ then the set $(V \star t) \cap H$ is empty for any $V \subset W$. Now we proceed as in the group case [22]. For $V \subset W$, we have $\mu \star f_{V}=\theta \star f_{V}$ off $U$. Hence,

$$
\int_{K \backslash U}\left(\mu \star f_{V}\right)(t) d m(t)=\int_{K \backslash U}\left(\theta \star f_{V}\right)(t) d m(t) \leq\|\theta\|\left\|f_{V}\right\|_{1} \leq \varepsilon a / 2 .
$$

By Chebyshev's inequality:

$$
m\left\{\left\{\mu \star f_{V}>a\right\} \cap(K \backslash U)\right\} \leq \varepsilon / 2
$$


and

$$
m\left\{\mu \star f_{V}>a\right\} \leq \varepsilon / 2+m(U)<\varepsilon .
$$

By [15, 5.1.B], we also get that if $g \in L^{1}(K),\left\{g \star f_{V}\right\}_{V \in \mathscr{U}}$ converges in $L^{1}$-norm to $g$. Since each $f_{V}$ is an element of $L^{2}(K)$ and $\mathscr{T}(K)$ is dense in $L^{2}(K)$ [24], we get the theorem.

Let us note that R. C. Vrem constructed in $L^{1}(K)$, with $K$ a compact hypergroup, approximate units satisfying the assertions (1), (2) and (4) of the Theorem 3.1. See [24].

\section{Lacunary sets for compact hypergroups}

Following [10, 16], we now define Riesz, nicely placed, Shapiro and Rosenthal sets. In the sequel, $K$ will be a compact hypergroup, $\Sigma$ its dual and $m$ the Haar measure on $K$.

Definition 4.1. A subset $\Lambda$ of $\Sigma$ is a Riesz set if every measure $\mu$ in $\mathscr{M}_{\Lambda}(K)$ is absolutely continuous with respect to the Haar measure of $K$.

Definition 4.2. Let $X$ be a closed subspace of $L^{1}$. The space $X$ is nicely placed if $B(X)$ is closed in $L(1, \infty)$. A subset $\Lambda$ of $\Sigma$ is nicely placed if $L_{\Lambda}^{1}(K)$ is nicely placed in $L^{1}(K)$.

We denote by $[\Lambda]$ the smallest nicely placed subset of $\Sigma$ containing $\Lambda$.

Definition 4.3. A subset $\Lambda$ of $\Sigma$ is a Shapiro set if every subset of $\Lambda$ is nicely placed.

Definition 4.4. A subset $\Lambda$ of $\Sigma$ is a Rosenthal set if $L_{\Lambda}^{\infty}(K)=\mathscr{C}_{\Lambda}(K)$.

It is known that every Rosenthal subset of a commutative discrete group is a Riesz set [16]. More generally, R. E. Dressler and L. Pigno have shown that the union of a Riesz set and of a Rosenthal set is a Riesz set [7]. G. Godefroy extended this result [11]; we generalize Godefroy's result to compact hypergroups.

Proposition 4.5. If $K$ is compact hypergroup and $\Sigma$ its dual, if $\Lambda \subset \Sigma$ is such that $\mathscr{M}_{\Lambda}=L_{\Lambda}^{1} \oplus\left(\mathscr{M}_{s}\right)_{\Lambda}$ and if $\Lambda_{0}$ is a Rosenthal set, then

$$
\mathscr{M}_{\Lambda \cup \Lambda_{0}}=L_{\Lambda_{1} \cup \Lambda_{2}}^{1} \oplus\left(\mathscr{M}_{S}\right)_{\Lambda}
$$

Let us remark that if $\Lambda$ is a Riesz set then $\left(\mathscr{M}_{s}\right)_{\Lambda}=\{0\}$ and $\Lambda \cup \Lambda_{0}$ is a Riesz set.

Proof. Let $\mu$ be in $\mathscr{M}_{\Lambda \cup \Lambda_{0}}(K)$ and consider $g_{n}=k_{n} \star \mu$ (where $\left(k_{n}\right)$ is 
an approximation of the identity in $\left.L^{1}(K)\right)$. We have,

$$
\begin{aligned}
\int_{K}\left(k_{n} \star \mu\right) f d m & =\iint_{k} k_{n}\left(x \star y^{-}\right) f(x) d \mu(y) d m(x) \\
& =\iint_{K} k_{n}^{-}\left(y \star x^{-}\right) f^{-}\left(x^{-}\right) d \mu(y) d m\left(x^{-}\right) \\
& =\iint_{K} k_{n}^{-}(x) f^{-}\left(y^{-} \star x\right) d \mu(y) d m(x) \\
& =\int_{K} k_{n}^{-}\left(\mu \star f^{-}\right) d m .
\end{aligned}
$$

We let $\bar{\Lambda}=\{\bar{\alpha}, \alpha \in \Lambda\}$ and $\Lambda^{\prime} \in \Sigma \backslash \bar{\Lambda}$. If $f \in L_{\Lambda^{\prime}}^{\infty}$ then $f^{-} \in L_{\Lambda}^{\infty}$ and $\mu \star f^{-} \in L_{\Lambda_{0}}^{\infty}=\mathscr{C}_{\Lambda_{0}}$. Therefore $\lim _{n \rightarrow+\infty}\left(\int g_{n} f d m\right)$ exists for every $f \in L_{\Lambda^{\prime}}^{\infty}$.

And now the proof proceeds exactly as in the commutative compact group case, see [11].

In the sequel, $K$ will be a commutative compact hypergroup and $\widehat{K}$ its dual. The second part of this section is devoted to the proof of the following result.

THEOREM 4.6. Let $K$ be a commutative compact hypergroup. Then every Shapiro subset of $\widehat{K}$ is a Riesz set.

Let $\mathscr{C} \subset \mathscr{P}(\widehat{K})$ be a family of subsets of $\widehat{K}$. We consider the class $\stackrel{\mathscr{C}}{=}=\{A \in \mathscr{P}(\widehat{K}):$ for all $B \subset A, B \in \mathscr{C}\}$. We have the following lemmas.

LEMMA 4.7. Let $K$ be a commutative compact hypergroup and $\mathscr{C}$ be a family of subsets of $\widehat{K}$. If every $\Lambda \in \mathscr{C}$ satisfies:

$$
\mu \in \mathscr{M}_{\Lambda}(K) \text { implies } \mu_{s} \in \mathscr{M}_{\Lambda}(K),
$$

then every $\Lambda \in \stackrel{\circ}{\mathscr{C}}$ is a Riesz set.

Proof. The proof here is similar to the group case [10, Lemma 1.1].

LEMMA 4.8. Let $K$ be a commutative compact hypergroup, $\Lambda$ be a subset of $\widehat{K}$ and $\mu \in \mathscr{M}_{\Lambda}(K)$. Then $\mu_{s} \in \mathscr{K}_{[\Lambda]}(K)$.

Proof. The proof proceeds as in the group case [10, Lemma 1.5]. Note that this proof does require Theorem 3.1.

Theorem 4.6 follows from these lemmas. 
REMARK. With the same arguments it can be shown that if $G$ is a compact group and $\Sigma$ its dual then every Shapiro subset of $\Sigma$ is a Riesz set.

A way to construct Riesz, nicely placed and Shapiro sets is the localization technique $[10,17]$. Let us introduce the following topology on $\widehat{K}$. For $\alpha \in \widehat{K}$, we say that $V_{\alpha}$ is a $\tau$-neighborhood of $\alpha$ if there exists a measure $\nu_{\alpha} \in D(K)$ such that

$$
\hat{\nu}_{\alpha}(\alpha) \neq 0, V_{\alpha} \supset \operatorname{spec} \nu_{\alpha}
$$

This defines a topology. Let us just mention that if $U_{\alpha}$ and $V_{\alpha}$ are two $\tau$-neighborhoods of $\alpha$ in $\widehat{K}$ then there exist two measures $\nu_{\alpha}$ and $\mu_{\alpha}$ in $D(K)$ such that

$$
\alpha \in U_{\alpha} \supset \operatorname{spec} \nu_{\alpha}, \alpha \in V_{\alpha} \supset \operatorname{spec} \mu_{\alpha} .
$$

We have $\nu_{\alpha} \star \mu_{\alpha} \in D(K)$ and $\alpha \in U_{\alpha} \cap V_{\alpha} \supset \operatorname{spec}\left(\nu_{\alpha} \star \mu_{\alpha}\right)$. Therefore $U_{\alpha} \cap V_{\alpha}$ is a $\tau$-neighborhood of $\alpha$. This topology corresponds to the Bohr topology in the commutative group case.

THEOREM 4.9. If $\Lambda$ is a subset of $\widehat{K}$ and if for every $\alpha \in \widehat{K}$ there exists $a$ $\tau$-neighborhood $V_{\alpha}$ of $\alpha$ such that

$$
\mu \in \mathscr{M}_{\Lambda \cap V_{\alpha}}(K) \text { implies } \mu_{s} \in \mathscr{M}_{\Lambda \cap V_{\alpha}}(K)
$$

then we also have

$$
\mu \in \mathscr{M}_{\Lambda}(K) \text { implies } \mu_{s} \in \mathscr{M}_{\Lambda}(K)
$$

Proof. Let $\Lambda \subset \widehat{K}, \alpha \notin \Lambda$ and $V_{\alpha}$ be a $\tau$-neighborhood of $\alpha$ satisfying (1) and (2). Let $\mu \in M_{\Lambda}(K)$. Then $\nu_{\alpha} \star \mu \in \mathscr{M}_{\Lambda \text { nspec } \nu_{\alpha}}(K)$ and $\nu_{\alpha} \star \mu \epsilon$ $\mathscr{M}_{\Lambda \cap V_{\alpha}}(K)$. Then $(\nu \star \mu)_{s} \in \mathscr{M}_{\Lambda \cap V_{\alpha}}(K)$. Since $\alpha \notin \Lambda, \alpha \notin \Lambda \cap V_{\alpha}$ and $\left(\widehat{\nu_{\alpha}} \widehat{\star}\right)_{s}(\alpha)=0$. Since $L^{1}(K)$ and $\mathscr{M}_{s}(K)$ are closed and invariant under convolutions by the elements of $D(K)$ [15], we have $\left(\nu_{\alpha} \star \mu\right)_{s}=\nu_{\alpha} \star \mu_{s}$ [17]. Thus $\widehat{\mu}_{s}(\alpha)=0$ and $\mu_{s} \in \mathscr{M}_{\Lambda}(K)$.

Let us notice that if $\Lambda$ is a subset of $\widehat{K}$ and if for every $\alpha \in \widehat{K}$ there exists a $\tau$-neighborhood $V_{\alpha}$ of $\alpha$ such that $\Lambda \cap V_{\alpha}$ is a Riesz set then $\Lambda$ is also a Riesz set.

THEOREM 4.10. If $\Lambda$ is a subset of $\widehat{K}$ and if for every $\alpha \in \widehat{K}$ there exists a $\tau$-neighborhood $V_{\alpha}$ of $\alpha$ such that $\Lambda \cap V_{\alpha}$ is a nicely placed set then $\Lambda$ is a nicely placed set.

Proof. We need two lemmas. 
LEMMA 4.11. Let $\nu \in D(K)$ and $\left(f_{n}\right)_{n \geq 1}$ be a bounded sequence in $L^{1}(K)$ which converges to 0 in $L(1, \infty)$. Then $\nu \star f_{n}$ belongs to $L^{1}(K)$ and there exists a subsequence $\left(\tilde{f}_{k}\right)_{k \geq 1}$ of $\left(f_{n}\right)$ such that the sequence

$$
\left(\nu \star \frac{1}{n} \sum_{k=1}^{n} \tilde{f}_{n}\right)_{n \geq 1}
$$

converges to 0 in $L(1, \infty)$.

Proof. By $[15,6.2 . \mathrm{B}]$, we have $\nu \star f_{n} \in L^{1}(K)$ and

$$
\left\|\nu \star f_{n}\right\|_{1} \leq\|\nu\|\left\|f_{n}\right\|_{1} .
$$

By [10, Lemma 1.2], there exists a subsequence $\left(\tilde{f}_{k}\right)$ of $\left(f_{n}\right)$ such that the sequence

$$
\left(\nu \star \frac{1}{n} \sum_{k=1}^{n} \tilde{f}_{k}\right)_{n \geq 1}
$$

converges in $L(1, \infty)$. It is easy to see that the assumption that the sequence $\left(f_{n}\right)$ converges to 0 in $L(1, \infty)$ implies that the sequence $\left(\nu \star \frac{1}{n} \sum_{k=1}^{n} \tilde{f}_{k}\right)_{n \geq 1}$ also converges to 0 in $L(1, \infty)$.

Lemma 4.12. Let $\Lambda$ be a subset of $\widehat{K}$ and $\alpha \notin \Lambda$. If there exists $a$ $\tau$-neighborhood $V_{\alpha}$ of $\alpha$ such that $\alpha \notin\left[V_{\alpha} \cap V\right]$ then $\alpha \notin[\Lambda]$.

Proof. Let $\nu_{\alpha} \in D(K)$ which satisfies (1). Let $f \in L_{\Lambda}^{1}(K)$. By Lemma 4.11, $\nu_{\alpha} \star f \in L^{1}(K)$. In fact, $\nu_{\alpha} \star f \in L_{V_{\alpha} \cap \Lambda}^{1}(K)$. Thus $\nu_{\alpha} \star L_{\Lambda}^{1}(K) \subset$ $L_{V_{a} \cap \Lambda}^{1}(K)$. Lemma 4.11 proves that if $X$ is nicely placed then $C=\{f$ : $\left.\nu_{\alpha} \star f \in X\right\}$ is also nicely placed: consider a sequence $\left(f_{n}\right)$ in $C$ which converges to $f$ in $L(1, \infty)$, then $\nu_{\alpha} \star f_{n}$ belongs to $X$ and $\frac{1}{n} \nu_{\alpha} \star \sum_{k=1}^{n} f_{k}$ also belongs to $X$. It follows by Lemma 4.11 that $f$ belongs to $C$. Then we have:

$$
\nu_{\alpha} \star L_{[\Lambda]}^{1}(K) \subset L_{\left[V_{\alpha} \cap \Lambda\right]}^{1}(K)
$$

Since $\nu_{\alpha} \star \alpha=\hat{\nu}_{\alpha}(\alpha) . \alpha$ and $\hat{\nu}_{\alpha}(\alpha) \neq 0,(3)$ shows that $\alpha \notin[\Lambda]$.

Let us now prove Theorem 4.10. Let $\alpha \notin \Lambda$ and $V_{\alpha}$ a $\tau$-neighborhood satisfying (1), then $\alpha \notin \Lambda \cap V_{\alpha}=\left[\Lambda \cap V_{\alpha}\right]$. And by Lemma 4.11, $\alpha \notin[\Lambda]$.

COROLLARY 4.13. If $\Lambda$ is a subset of $\widehat{K}$ and if for every $\alpha \in \widehat{K}$ there exists a $\tau$-neighborhood $V_{\alpha}$ of $\alpha$ such that $\Lambda \cap V_{\alpha}$ is a Shapiro set then $\Lambda$ is a Shapiro set. 
COROLLARY 4.14. Let $\Lambda_{1}$ be a nicely placed subset of $\widehat{K}$ and $\Lambda_{2}$ a $\tau$ closed subset of $\widehat{K}$. Then the union $\Lambda_{1} \cup \Lambda_{2}$ is nicely placed. In particular, every $\tau$-closed subset is nicely placed.

This extends a result of Y. Meyer [17]. We also get an extension of the Mooney-Havin theorem [13, 18].

Corollary 4.15. Let $\Lambda_{1}$ be a nicely placed subset of $\widehat{K}$ and $\Lambda_{2}$ be a $\tau$-closed subset of $\widehat{K}$. Then the space $L^{1} / L_{\Lambda_{1} \cup \Lambda_{2}}^{1}(K)$ is weakly sequentially complete.

Proof. The result follows from Corollary 4.14 and from the fact that if $\Lambda$ is a nicely placed subset of $\widehat{K}$ then the space $L^{1} / L_{\Lambda}^{1}(K)$ is weakly sequentially complete [10].

\section{Examples}

\section{Conjugacy classes of compact non-commutative groups.}

Let $G$ be a compact non-commutative group, with normalized Haar measure $\sigma$ and $\Sigma$ be its dual object. We say that a subset $\Lambda$ of $\Sigma$ is central Riesz if every central measure $\mu$ in $\mathscr{M}_{\Lambda}(G)$ is absolutely continuous with respect to the Haar measure of $G$. Central nicely placed and central Shapiro subsets of $\Sigma$ are defined in the same way. For $x \in G$, let $x^{G}=\left\{t^{-1} x t, t \in G\right\}$ the conjugacy class of $x$. Let $K=\left\{x^{G}, x \in G\right\}$ have the quotient topology. The space $K$, with the operation

$$
\delta_{x^{G}} \star \delta_{y^{G}}=\int_{G} \delta_{\left(t^{-1} x t y\right)^{G}} d \sigma(t)
$$

is a compact commutative hypergroup [15]. Each $\tau \in \Sigma$ has a representation space of finite dimension $d_{\tau}$ and trace $\chi_{\tau}$. The functions $\chi_{\tau}$ are called characters but the hypergroup characters are normalized by dividing $\chi_{\tau}$ by $d_{\tau}$. More precisely, if $\pi: G \rightarrow K$ is the natural mapping then $\psi_{\tau}$ on $K$ is defined by: $\psi_{\tau} \circ \pi=d_{\tau}^{-1} \chi_{\tau}$ and $\widehat{K}=\left\{\psi_{\tau}, \tau \in \Sigma\right\}$. The Haar measure $m$ on $K$ is induced from the Haar measure on $G . \widehat{K}$ is a commutative discrete hypergroup. The functions defined on $K$ (respectively the measures of $K$ ) correspond to the central functions defined on $G$ (respectively the central measures of $G$ ). It follows that Riesz (respectively nicely placed) subsets of $\widehat{K}$ correspond to central Riesz (respectively central nicely placed) subsets of $\Sigma$. Let us now consider two examples: 
a) The special unitary group $S U(2)$ consists of all $2 \times 2$ matrices $\left(\begin{array}{c}\alpha-\beta \\ -\bar{\beta}\end{array}\right)$ where $\alpha, \beta \in \mathbb{C}$ and $|\alpha|^{2}+|\beta|^{2}=1$. Following [14] we now recall the construction of the dual $\Sigma$ of $S U(2)$. Let $l$ be a number in the set $\left\{0, \frac{1}{2}, 1\right.$, $\left.\frac{3}{2}, \ldots\right\}$. We construct a representation of $S U(2)$ of dimension $2 l+1$ as follows.

Let $H_{l}$ be the linear space of all complex one variable polynomials of degree not exceeding $2 l$. Let $u=\left(\begin{array}{c}\alpha-\beta \\ -\bar{\beta}\end{array}\right)$. For all $f \in H_{l}$, we define:

$$
\left(T_{u}^{(l)} f\right)(z)=(\beta z+\bar{\alpha})^{2 l} f\left(\frac{\alpha z-\bar{\beta}}{\beta z+\bar{\alpha}}\right) .
$$

The mapping $T^{(l)}: u \rightarrow T_{u}^{(l)}$ is a $(2 l+1)$-dimensional representation of $S U(2)$ and the set $\left\{T^{(0)}, T^{\left(\frac{1}{2}\right)}, T^{(1)}, \ldots\right\}$ is a complete set of continuous unitary irreducible representations of $S U(2)$. That is, for each $n=$ $1,2,3, \ldots, \Sigma$ contains exactly one element of dimension $n$. We write its character by $\chi_{n}$. We now describe the hypergroup $K$ of conjugacy classes of $S U(2)$, see [15] for more details. We identify $K$ with $[0, \pi]$ where $\theta$ in $[0, \pi]$ corresponds to the conjugacy class containing the matrix

$$
\left(\begin{array}{cc}
e^{i \theta} & 0 \\
0 & e^{-i \theta}
\end{array}\right)
$$

The hypergroup character $\psi_{n}$ on $K$ corresponding to $\chi_{n}$ is equal to

$$
\left.\psi_{n}(\theta)=\frac{\sin n \theta}{n \sin \theta}, \theta \in\right] 0, \pi\left[; \psi_{n}(0)=\psi_{n}(\pi)=1 .\right.
$$

The Haar measure $m$ on $K$ is given by

$$
\int_{K} f d m=\frac{2}{\pi} \int_{0}^{\pi} f(\theta) \sin ^{2} \theta d \theta
$$

We now give some examples of $\tau$-closed subsets of $\widehat{K}$. A subset $F$ of $\widehat{K}$ is $\tau$-closed if there exists a measure $\nu$ in $D(K)$ such that

$$
F=\left\{\psi_{n}, n \in \mathbb{N}^{\star}, \hat{\nu}\left(\psi_{n}\right)=0\right\}
$$

(1) Let $r \in \mathbb{N}^{\star} \backslash\{1\}$. Then the set $\left\{\psi_{k r}, k \in \mathbb{N}^{\star}\right\}$ is $\tau$-closed. Indeed, take $\nu=\delta_{\pi / r}$. Then $\hat{\nu}\left(\psi_{n}\right)=(\sin n \pi / r) /(n \sin \pi / r)=0$ if and only if $n$ is a multiple of $r$ in $\mathbb{Z}$.

(2) Let $r \in \mathbb{N}^{\star}$. Then the set $\left\{\psi_{(2 k+1) r}, k \in \mathbb{N}\right\}$ is $\tau$-closed. Consider the discrete measure $\nu=\sin \theta_{1} \delta_{\theta_{1}}-\sin \theta_{2} \delta_{\theta_{2}}$, with $\theta_{1}=\pi / r-\pi / \sqrt{2}$ and $\theta_{2}=\pi / \sqrt{2}$. 
Then,

$$
\begin{aligned}
\hat{\nu}\left(\psi_{n}\right) & =\frac{1}{n}(\sin n(\pi / r-\pi / \sqrt{2})-\sin n \pi / \sqrt{2}) \\
& =\frac{2}{n} \sin n(\pi / 2 r-\pi / \sqrt{2}) \cos n \pi / 2 r
\end{aligned}
$$

and

$$
\begin{array}{ll}
\left(\hat{\nu}\left(\psi_{n}\right)=0\right) \quad \text { if and only if }(\cos n \pi / 2 r=0) \\
& \text { if and only if }(n \in\{(2 k+1) r, k \in \mathbb{Z}\}) .
\end{array}
$$

b) C. F. Dunkl and D. E. Ramirez constructed in [8] an interesting family of compact countable hypergroups $H_{a}$, for $0<a \leq \frac{1}{2} . H_{a}$ has the topological structure of the one-point compactification of the nonnegative integers. The case $H_{1 / p}, p$ prime, is the set of equivalence classes of the $p$-adic integers modulo the group of units (under multiplication). The hypergroup $H_{a}$ is identified with $\{0,1,2, \ldots \infty\}$. The invariant measure $m$ is given by

$$
\begin{gathered}
m(\{k\})=a^{k}(1-a) \text { for } k<\infty, m(\{\infty\})=0 . \\
\widehat{H}_{a}=\left\{\psi_{0}, \psi_{1}, \psi_{2}, \ldots\right\} \text { where } \psi_{0} \equiv 1 \text { and } \\
\psi_{n}(k)= \begin{cases}1 & \text { for } k \geq n \\
a /(a-1) & \text { for } k=n-1, n \geq 1 \\
0 & \text { for } k<n-1 .\end{cases}
\end{gathered}
$$

The following sets are $\tau$-clopen:

(1) $\left\{\psi_{p}, \psi_{p+1}, \psi_{p+2}, \ldots\right\}$ for all $p \geq 2$.

(Take $\nu=\delta_{p-2}$ )

(2) $\left\{\psi_{p}\right\}$ for all $p \in \mathbb{N}$. (Take $\nu=\left((a+1) / a \delta_{p-1}-\delta_{p}\right)+$

$\sum_{j=0}^{\infty} 1 / 2^{j}\left(\delta_{p+2 j+1}-\delta_{p+2 j+2}\right)$ for $p \geq 1$ and $\nu=$

$\sum_{j=0}^{\infty} 1 / 2^{j}\left(\delta_{2 j}-\delta_{2 j+1}\right)$ for $\left.p=1\right)$.

(3) $\left\{\psi_{0}, \psi_{1}, \ldots, \psi_{p}\right\}$ for all $p \in \mathbb{N}$.

(Take $\left.\nu=\sum_{j=0}^{\infty} 1 / 2^{j}\left(\delta_{p+2 j}-\delta_{p+2 j+1}\right)\right)$.

\section{6. $\Lambda(1)$-sets in compact hypergroups}

It is known that if $G$ is a compact commutative group and $\Lambda$ a subset of its dual then we have the following equivalences.

(1) $\Lambda$ is a $\Lambda(1)$-set if and only if $L_{\Lambda}^{1}(G)$ is reflexive [1]

(2) $\Lambda$ is a Riesz set if and only if $L_{\Lambda}^{1}(G)$ has Radon-Nikodym property [16]. 
Let us recall that a Banach space $X$ has the Radon-Nikodym property (RNP) if and only if every linear continuous operator

$$
T: L^{1}(\Omega, \mathscr{A}, \mu) \rightarrow X
$$

(where $\mu$ is a probability measure) is representable by a $X$-valued strongly $\mu$-measurable and bounded function $F$ that is for all $\varphi \in L^{1}(\Omega, \mathscr{A}, \mu)$,

$$
T(\varphi)=\int \varphi(\omega) F(\omega) d \mu(\omega) .
$$

If $X$ is a reflexive Banach space then $X$ has RNP [6]. If follows that a $\Lambda(1)$-set is a Riesz set. Our aim is to extend the results (1) and (2) to compact hypergroups. In the following $K$ will denote a compact not necessarily commutative hypergroup, $m$ its Haar measure and $\Sigma$ its dual.

Definition 6.1. Let $0<p<\infty$. A subset $\Lambda$ of $\Sigma$ is called $\Lambda(p)$-set if for some $0<q<p$ there exists a constant $C$ such that

$$
\|f\|_{p} \leq C\|f\|_{q} \text {, for all } f \in \mathscr{T}_{\Lambda}(K) .
$$

$\Lambda$ is called central $\Lambda(p)$ if (4) holds for all $f$ in $\mathscr{T}_{\Lambda}(K)$ which are central.

Proposition 6.2. Let $\Lambda$ be a subset of $\Sigma$. Then $\Lambda$ is $\Lambda(1)$-set if and only if $L_{\Lambda}^{1}(K)$ is reflexive.

Proof. Suppose that $\Lambda$ is a $\Lambda(1)$-set of $\Sigma$. Then for some $0<q<1$ there exists a constant $C$ such that

$$
\|f\|_{1} \leq C\|f\|_{q}, \text { for all } f \in \mathscr{T}_{\Lambda}(K) .
$$

Let $\left(F_{\alpha}\right)$ be an approximate unit satisfying Theorem 3.1. Let $f \in L_{\Lambda}^{1}(K)$ then $F_{\alpha} \star f \in \mathscr{T}_{\Lambda}(K)$ and, for $0<q<1$, we have

$$
\left\|F_{\alpha} \star f\right\|_{1} \leq C\left\|F_{\alpha} \star f\right\|_{q} .
$$

Let $\varepsilon>0$ and $\alpha$ be such that

$$
\left\|F_{\alpha} \star f-f\right\|_{1} \leq \varepsilon .
$$

Then,

$$
\begin{aligned}
\|f\|_{1} & \leq\left\|F_{\alpha} \star f\right\|_{1}+\left\|f-F_{\alpha} \star f\right\|_{1} \leq C\left\|F_{\alpha} \star f\right\|_{q}+\varepsilon \\
& \leq C\left[\left\|F_{\alpha} \star f-f\right\|_{q}^{q}+\|f\|_{q}^{q}\right]^{1 / q}+\varepsilon \leq C\left[\left\|F_{\alpha} \star f-f\right\|_{1}^{q}+\|f\|_{q}^{q}\right]^{1 / q}+\varepsilon \\
& \leq C\left(\varepsilon^{q}+\|f\|_{q}^{q}\right)^{1 / q}+\varepsilon .
\end{aligned}
$$

We have proved that there exists a constant $C$ depending only on $q$ such that for all $f \in L_{\Lambda}^{1}(K)$, we have

$$
\|f\|_{1} \leq C\|f\|_{q}
$$


that is, the $L^{1}$ and $L^{q}$ topologies coincide on $L_{\Lambda}^{1}(K)$ and $L_{\Lambda}^{1}(K)$ is reflexive. For the converse, see [1].

Proposition 6.3. Let $\Lambda$ be a subset of $\Sigma$. Then $\Lambda$ is Riesz if and only if $L_{\Lambda}^{1}(K)$ has $R N P$.

Proof. Suppose first that $\Lambda$ is Riesz. The space $L_{\Lambda}^{1}(K)$ has RNP if and only if its separable subspaces have RNP [6]. Let $S$ be a separable subspace of $L_{\Lambda}^{1}(K)$, then $S \subset L_{\Lambda^{\prime}}^{1}(K)$ with $\Lambda^{\prime} \subset \Lambda$ and $\Lambda^{\prime}$ countable. Since $L_{\Lambda}^{1}(K)=\mathscr{M}_{\Lambda}(K), L_{\Lambda^{\prime}}^{1}(K)$ is a separable dual. On the other hand, suppose that $L_{\Lambda}^{1}(K)$ has RNP. Let $\mu \in \mathscr{M}_{\Lambda}(K), f \in L^{1}(K), \theta \in \mathscr{C}(K)$ then

$$
\begin{aligned}
\langle f \star \mu, \theta\rangle & =\int_{K}(f \star \mu) \theta d m=\int_{K} f\left(\theta \star \mu^{-}\right) d m[15,6.2 \mathrm{D}] \\
& =\int_{K} f(g) \int_{K} \theta\left(g \star y^{-}\right) d \mu^{-}(y) d m(g) \\
& =\int_{K} \int_{K} \int_{K} f(g) \theta(s) d\left(\delta_{g} \star \delta_{y^{-}}\right)(s) d \mu^{-}(y) d m(g) .
\end{aligned}
$$

Also,

$$
\begin{aligned}
\int_{K} f(g)\left\langle\delta_{g} \star \mu, \theta\right\rangle d m(g) & =\int_{K} f(g) \int_{K} \theta(x) d\left(\delta_{g} \star \mu\right)(x) \\
& =\int_{K} \int_{K} f(g) \theta_{g}(y) d \mu(y)[15,3.1 . \mathrm{F}] \\
& =\int_{K} \int_{K} \int_{K} f(g) \theta(s) d\left(\delta_{g} \star \delta_{y}\right)(s) d \mu(y) d m(g)
\end{aligned}
$$

and

$$
\langle f \star \mu, \theta\rangle=\int_{K} f(g)\left\langle\delta_{g} \star \mu, \theta\right\rangle d m(g) .
$$

Let $T_{\mu}$ be the operator from $L^{1}(K)$ into $L_{\Lambda}^{1}(K)$ defined by $T_{\mu}(f)=f \star \mu$. $L_{\Lambda}^{1}(K)$ has RNP then the function $g \rightarrow \delta_{g} \star \mu$ is almost everywhere $L_{\Lambda}^{1}(K)$ valued. Thus $\mu$ is in $L_{\Lambda}^{1}(K)$.

Let $G$ be a compact group, $\Sigma$ its dual and $\Lambda$ a subset of $\Sigma$. Let us denote by $L_{\Lambda}^{1 C}(G)$ the subspace of central functions of $L_{\Lambda}^{1}(G)$. RNP and reflexivity are isomorphic properties therefore by Proposition 6.2 and Proposition 6.3, we get

CoRollary 6.4. (1) $\Lambda$ is a central $\Lambda(1)$-set if and only if $L_{\Lambda}^{1 C}(G)$ is reflexive. 
(2) $\Lambda$ is a central Riesz set if and only if $L_{\Lambda}^{1 C}(G)$ has $R N P$.

COROLlary 6.5. If $\Lambda$ is a central $\Lambda(1)$-set then $\Lambda$ is a central Riesz set.

This answers a question of R. G. M. Brummelhuis [5].

REMARK. In the non-commutative group case, central $\Lambda(p)$-sets are more abundant than $\Lambda(p)$-sets, see [5]. For example for all $n \geq 2$, the dual object of $S U(n)$ does not contain an infinite $\Delta(p)$-set for any $p>0([19,20])$.

\section{Shapiro sets for compact groups whose center contains the circle}

G. Godefroy proved the following result [10]. Let $\Gamma$ be a totally ordered discrete group and $\Lambda$ be a subset of $\Gamma$ such that

$$
\Lambda \cap\left\{\alpha^{\prime} \leq \alpha\right\} \text { is a } \Lambda(1) \text {-set for every } \alpha \in \Gamma .
$$

Then $\Lambda$ is a Shapiro set of $\Gamma$.

We generalize this result to compact groups whose center contains a circle group and precise a result of R. G. M. Brummelhuis [5]. Examples of such groups are the unitary group $U(n)$, isotropy groups of bounded symmetric domains. In this section $G$ will denote a compact metrizable group whose center contains a circle group. We denote by $\Sigma$ its dual and by $m$ its Haar measure. For $\tau$ in $\Sigma$, let $H(\tau)$ denote the representation space of $\tau$ and $d_{\tau}$ the dimension of $H(\tau)$. If $\chi_{\tau}$ denotes the character of $\tau$ then for $F \in \mathscr{T}(G)$ and $g \in G$

$$
F(g)=\sum_{\tau \in \Sigma} d_{\tau}\left(\chi_{\tau} \star F\right)(g)=\sum_{\tau \in \Sigma} d_{\tau} \operatorname{tr}\{\widehat{F}(\tau) \tau(g)\}
$$

where tr means trace.

Fix an injective homomorphism $\left.\left.\mathbb{T}=\left\{e^{i \theta}, \theta \in\right]-\pi, \pi\right]\right\} \hookrightarrow Z(G)$, the center of $G ; e^{i \theta}$ will denote an element of $\mathbb{T}$ as well as an element of $Z(G)$. By Schur's lemma there exists for each $\tau$ in $\Sigma$ a unique $n(\tau) \in \mathbb{Z}$ such that

$$
\tau\left(e^{i \theta}\right)=e^{i n(\tau) \theta} I d_{H(\tau)}, \text { for all } e^{i \theta} \in \mathbb{T} .
$$

If $f$ is a function on $G$ and $g$ in $G$, the "slice" function $f_{g}$ on $\mathbb{T}$ is defined by:

$$
f_{g}\left(e^{i \theta}\right)=f\left(e^{i \theta} g\right) \text {. }
$$

For $f$ in $\mathscr{T}(G), f_{g} \in \mathscr{T}(\mathbb{T})$ and

$$
f_{g}\left(e^{i \theta}\right)=\sum_{m \in \mathbf{Z}} \pi_{m} f(g) e^{i m \theta}
$$


where the projections $\pi_{m}$ are defined by

$$
\pi_{m} f(g)=\sum_{\substack{\tau \in \Sigma \\ n(\tau)=m}} d_{\tau} \operatorname{tr}[\hat{f}(\tau) \tau(g)] .
$$

Define the projection $P_{N}$ on $\mathscr{T}(G)$ by

$$
P_{N}(f)=\sum_{m \leq N} \pi_{m} f
$$

The following lemma follows from [5].

Lemma 7.1. For all $p, 0<p<1$, there exists a constant $C$ such that for all $f$ in $\mathscr{T}(G)$,

$$
\left\|P_{N} f\right\|_{p} \leq C\|f\|_{1}
$$

We need the following lemma

LEMMA 7.2. Let $\Lambda \subset \Sigma, f \in L^{1}(G)$ and $n(\Lambda)=\{n(\tau), \tau \in \Lambda\}$. If $f$ belongs to $L_{\Lambda}^{1}(G)$ then for almost every $g \in G, f_{g}$ belongs to $L_{n(\Lambda)}^{1}(\mathbb{T})$.

Proof. There exists a sequence $\left(f^{(n)}\right)_{n \geq 1}$ of trigonometric polynomials in $\mathscr{T}_{\Lambda}(G)$ such that $\left(\left\|f^{(n)}\right\|_{1}\right)_{n \geq 1}$ is bounded and $\left(f^{(n)}\right)_{n \geq 1}$ converges to $f$ in $L^{1}$-norm.

Up to a subsequence, we may assume that $M=\int_{G} \sum_{n}\left|f^{(n)}(g)-f(g)\right| d m(g)$ is finite; by invariance we get that, for all $e^{i \theta}$ in $\mathbb{T}$,

$$
M=\int_{G} \sum_{n}\left|f^{(n)}\left(g e^{i \theta}\right)-f\left(g e^{i \theta}\right)\right| d m(g) .
$$

So

$$
\int_{\mathrm{T}} \int_{G} \sum_{n}\left|f^{(n)}\left(g e^{i \theta}\right)-f\left(g e^{i \theta}\right)\right| d m(g) \frac{d \theta}{2 \pi} \text { is finite. }
$$

Hence, for almost every $g$ in $G, \int_{\mathbb{T}} \sum_{n}\left|f^{(n)}\left(g e^{i \theta}\right)-f\left(g e^{i \theta}\right)\right| d \theta / 2 \pi$ is finite. So that, for almost every $g$ in $G,\left(f_{g}^{(n)}\right)_{n \geq 1}$ converges to $f_{g}$ in $L^{1}(\mathbb{T})$. Since $f_{g}^{(n)} \in L_{n(\Lambda)}^{1}(\mathbb{T})$, it follows that $f_{g} \in L_{n(\Lambda)}^{1}(\mathbb{T})$.

Let us remark that if, for almost every $g \in G$, the slice function $f_{g}$ belongs to $L_{n(\Lambda)}^{1}(\mathbb{T})$ then the function $f$ belongs to $L_{\widetilde{\Lambda}}^{1}(G)$ where $\tilde{\Lambda}=\{\tau \in$ $\Sigma, n(\tau)=n(\beta)$, for some $\beta \in \Lambda\}$. We are now ready to prove the main result of this section. 
THEOREM 7.3. Let $G$ be a compact group whose center contains a copy of the circle group. Let $\Lambda$ be a subset of $\Sigma$ such that

$$
\{\tau \in \Lambda, n(\tau) \leq N\} \text { is a } \Lambda(1) \text {-set for every } N \in \mathbb{Z} .
$$

Then $\Lambda$ is a Shapiro subset of $\Sigma$.

Proof. Since (7) is hereditary, it is enough to prove that $\Lambda$ is nicely placed. Let $\left(f_{n}\right)_{n \geq 1}$ be a sequence in $B\left(L_{\Lambda}^{1}\right)$ which converges to $f$ in $L(1, \infty)$, and let $\tau$ be in $\Sigma \backslash \Lambda$; we have to prove that $\hat{f}(\tau)=0$.

Let $\alpha \in \Sigma$ be such that $n(\alpha) \geq n(\tau)$. Consider the projection $P_{n(\alpha)}$ defined by (6). We let $\Gamma_{n(\alpha)}=\{\delta \in \Sigma, n(\delta)>n(\alpha)\}$. If supp $\hat{f} \subset \Lambda$ then $P_{n(\alpha)} f \in L_{\Lambda \backslash \Gamma_{n(\alpha)}}^{1}$. Since $\Lambda \backslash \Gamma_{n(\alpha)}$ is a $\Lambda(1)$-set, there exists $K>0$ such that for every $f$ in $L_{\Lambda}^{1}$,

$$
\left\|P_{n(\alpha)} f\right\|_{1} \leq K\left\|P_{n(\alpha)} f\right\|_{1 / 2} .
$$

Also by Lemma 7.1, $\left\|P_{n(\alpha)} f\right\|_{1 / 2} \leq C\|f\|_{1}$. Thus $P_{n(\alpha)}$ is $\|\cdot\|_{1}$-continuous from $L_{\Lambda}^{1}$ into $L_{\Lambda \backslash \Gamma_{n(\alpha)}}^{1}$ and $L_{\Lambda}^{1}=L_{\Lambda \backslash \Gamma_{n(\alpha)}}^{1} \oplus L_{\Lambda \cap \Gamma_{n(\alpha)}}^{1}$. Let $\left(f_{n}^{\prime}\right)$ be a subsequence of $\left(f_{n}\right)$ which converges almost everywhere. Let $g_{n}^{\prime}=P_{n(\alpha)}\left(f_{n}^{\prime}\right)$ and $h_{n}^{\prime}=f_{n}^{\prime}-g_{n}^{\prime}$. The sequences $\left(g_{n}^{\prime}\right)$ and $\left(h_{n}^{\prime}\right)$ are $\|\cdot\|_{1}$-bounded and thus by Lemma $1.2[10]$ there exist subsequences $\left(g_{n}^{\prime \prime}\right)$ and $\left(h_{n}^{\prime \prime}\right)$, indexed by the same set, which converge in Cesaro mean almost everywhere and in $L(1, \infty)$ to $g$ and $h$, respectively. We have $f=g+h$.

Since $\Lambda \backslash \Gamma_{n(\alpha)}$ is a $\Lambda(1)$-set, the space $L_{\Lambda \backslash \Gamma_{n(\alpha)}}^{1}$ is $L(1, \infty)$ closed and thus $g \in L_{\Lambda \backslash \Gamma_{n(\alpha)}}^{1}$ and $\hat{g}(\tau)=0$. It remains to show that $\hat{h}(\tau)=0$. The sequence $k_{n}=\frac{1}{n} \sum_{j=1}^{n} h_{j}^{\prime \prime}$ converges to $h$ in $L(1, \infty)$ and is bounded in $L_{\Lambda \cap \Gamma_{n(\alpha)}}^{1}$. In particular $k_{n}$ is in $L_{\Gamma_{n(\alpha)}}^{1}$. Thus we have to show that $L_{\Gamma_{n(\alpha)}}^{1}$ is nicely placed.

In fact, it suffices to prove that $L_{\Gamma^{+}}^{1}$ is nicely placed where $\Gamma^{+}=\{\alpha \in \Sigma$, $n(\alpha)>0\}$. Let $\left(f^{(n)}\right)$ be a sequence in $B\left(L_{\Gamma^{+}}^{1}\right)$ which converges to $f$ in $L(1, \infty)$. We have to show that $\hat{f}(\alpha)=0$ for $\alpha \notin \Gamma^{+}$.

Up to a subsequence we may suppose that $\left(f^{(n)}\right)$ converges to $f$ a.e. And for almost every $g \in G, e^{i \theta} \in \mathbb{T}, f_{g}^{(n)}\left(e^{i \theta}\right)$ tends to $f_{g}\left(e^{i \theta}\right)$. By Lemma 7.2, we have that $f_{g}^{(n)} \in L_{n\left(\Gamma^{+}\right)}^{1}$ and $\left(\left\|f_{g}^{(n)}\right\|_{1}\right)$ is bounded. Since $n\left(\Gamma^{+}\right)$is nicely placed, $f_{g} \in L_{n\left(\Gamma^{+}\right)}^{1}$ for almost every $g$ and by the remark after Lemma 7.2 $f \in L_{\Gamma^{+}}^{1}$. This proves the theorem.

Our result improves Theorem 2.1 of [5]. 
We now give some applications of this theorem.

First, we give a non commutative extension of the Mooney-Havin theorem $([13,18])$.

COROLLARY 7.4. Let $G$ be a compact group whose center contains a copy of circle group. Let $\Lambda$ be a subset of $\Sigma$ satisfying (7) then the space $L^{1} / L_{\Lambda}^{1}(G)$ is weakly sequentially complete.

Proof. The result follows from Theorem 7.3 and Lemma 1.8 of [10].

We are now interested in the "central version" of Theorem 7.3. Let us note that if $\left(f_{n}\right)$ is a bounded sequence in $L_{\Lambda}^{1 C}(G)$ which converges to $f$ in $L(1, \infty)$ then $f$ is also a central function. And if $f$ is a central trigonometric polynomial then the projection $P_{N} f$ is also a central function.

THEOREM 7.5. Let $G$ be a compact group whose center contains a copy of the circle group. Let $\Lambda$ be a subset of $\Sigma$ such that

$$
\{\tau \in \Lambda, n(\tau) \leq N \text { is a central } \Lambda(1) \text {-set for every } N \in \mathbb{Z} .
$$

Then $\Lambda$ is a central Shapiro subset of $\Sigma$.

Let us note that under these assumptions $\Lambda$ is central Riesz. Another easy consequence of the theorem is the following corollary.

COROLlARY 7.6. Let $G$ be a compact group whose center contains a copy of the circle group. Let $\Lambda$ be a subset of $\Sigma$ such that

(1) For each $m \in \mathbb{Z}$ the set $\{\tau \in \Lambda: n(\tau)=m\}$ is a $\Lambda$ (1)-set.

(2) The set $\{n(\tau): \tau \in \Lambda\}$ is bounded from below.

Then $\Lambda$ is a Shapiro subset of $\Sigma$.

Let us mention that under these assumptions R. G. M. Brummelhuis proved that $\Lambda$ is a Riesz subset of $\Sigma$ [3].

REMARK. Let $G$ be a compact group and $\Lambda$ be a subset of its dual. We denote by $\widetilde{\Lambda}$ the set of $\alpha$ 's in $\Lambda$ such that the restriction of $F_{\alpha}: f \rightarrow \hat{f}(\alpha)$ to $B\left({ }_{\Lambda}^{1 C}(G)\right)$ is $L^{p}$-continuous $(0<p<1)$. Then we can show that for every central Shapiro set $\Lambda$, one has $\Lambda=\tilde{\Lambda}$. We can also give another "central" extension of Theorem 3.2 of [3].

Proposition 7.7. Let $G$ be a compact group whose center contains a copy of the circle group. Let $\Lambda$ be a subset of $\Sigma$ such that

(1) For each $m \in \mathbb{Z}$ the set $\{\tau \in \Lambda, n(\tau)=m\}$ is central Shapiro.

(2) The set $\{n(\tau), \tau \in \Lambda\}$ is bounded from below.

Then $\Lambda$ is a central Ries $z$ subset of $\Sigma$. 
Proof. Let $\mu$ be in $\mathscr{M}_{\Lambda}^{C}(G)$ and $\left(F_{n}\right)$ be a sequence of central trigonometric polynomials satisfying Theorem 3.1. Then $F_{n} \star \mu$ belongs to $\mathscr{T}_{\Lambda}^{C}(G)$ and $\mu_{a}$ belongs to the set $C$ which is the closure of the set $\|\mu\| B\left(\mathscr{T}_{\Lambda}^{C}\right)$ in $\|\cdot\|_{p}(0<p<1)$. Then for all $F \in B\left(\mathscr{T}^{C}(G)\right), F \star \mu_{a}$ also belongs to $C$. Following [3] we consider for $m \in \mathbb{Z}$ the projection $\pi_{m}: \mathscr{T}(G) \rightarrow \mathscr{T}(G)$ defined by

$$
\left(\pi_{m} f\right)(k)=\int_{-\pi}^{\pi} f\left(e^{i \theta} k\right) e^{-i m \theta} d \theta / 2 \pi=\sum_{n(\tau)=m} d_{\tau}\left(\chi_{\tau} \star f\right)(k) .
$$

Since the set $\{n(\tau), \tau \in \Lambda\}$ is bounded from below, $\pi_{m}$ is $L^{p}$-continuous on $\mathscr{T}_{\Lambda}(G)(0<p<1)$ [3]. Let $\left(f_{n}\right)$ be a sequence in $\mathscr{T}_{\Lambda}^{C}(G)$ with $\left(\left\|f_{n}\right\|_{1}\right)$ bounded and let $f$ be in $\mathscr{T}(G)$ such that $\left(f_{n}\right)$ converges to $f$ in $\|\cdot\|_{p}$ $(0<p<1)$ then $\left(\pi_{m}\left(f_{n}\right)\right)$ converges to $\pi_{m}(f)$ in $\|\cdot\|_{p}$. Moreover we have that $\operatorname{spec} \pi_{m}(f) \subset\{\tau \in \Lambda, n(\tau)=m\}$ since the set $\{\tau \in \Lambda, n(\tau)=m\}$ is central Shapiro. Therefore $f$ belongs to $\mathscr{T}_{\Lambda}^{C}(G)$. It follows that for all $F \in \mathscr{T}^{C}(G), F \star \mu_{a}$ belongs to $\mathscr{T}_{\Lambda}^{C}(G)$ and,

$$
\text { for all } F \in \mathscr{T}^{C}(G), F \star \mu_{s} \in \mathscr{T}_{\Lambda}^{C}(G) \text {. }
$$

For each $\sigma \in \Lambda$ and each $k \in G$, consider the linear functional:

$$
f \rightarrow d_{\sigma}\left(\chi_{\sigma} \star f\right)(k) \text {. }
$$

Let us denote by $Y$ the space $T_{\{\tau \in \Lambda, n(\tau)=n(\sigma)\}}^{C}$. Let $\left(f_{n}\right)$ and $f$ be in $Y$ such that $\left(\left\|f_{n}\right\|_{1}\right)$ is bounded and $\left(f_{n}\right)$ converges to $f$ in $\|\cdot\|_{p} \quad(0<p<1)$. We have that $\left(\hat{f}_{n}(\tau)\right)$ converges to $\hat{f}(\tau)$ for $\tau \in\{\tau \in \Lambda, n(\tau)=n(\sigma)\}$ since this set is central Shapiro. And $\left(\left(\chi_{\sigma} \star f_{n}\right)(k)=\operatorname{tr}\left(\hat{f}_{n}(\sigma) \sigma(k)\right)\right.$ converges to $\left(\chi_{\sigma} \star f\right)(k)$. Hence the functionals $(9)$ are $L^{p}$-continuous on the bounded sets of $Y$. From the $L^{p}$-continuity of the projection $\pi_{n(\sigma)}$ it follows that

(10) functionals (9) are $L^{p}$-continuous on bounded subsets of $\mathscr{T}_{\Lambda}^{C}(G)$.

(8) and (10) imply that $\mu_{s}=0$ (see [3] for more details).

We give some applications to the unit sphere in $\mathbb{C}^{n}$, homogeneous spaces and bounded symmetric domains.

Let $S=S_{2 n-1}=\left\{z \in \mathbb{C}^{n},|z|=1\right\}$ be the unit sphere in $\mathbb{C}^{n} . S_{1}$ is just the unit circle $\mathbb{T} . S_{3}$ can be identified with $S U(2)$ and we can then apply the results of Section 5. For $n>2, S_{2 n-1}$ does not have the structure of a 
compact group. We will extend the results of Section 7 to $S_{2 n-1}$ and more generally to homogeneous spaces.

Let us recall some basic facts about $S_{2 n-1}$ [3]. For non-negative integers $p$ and $q$, we denote by $H(p, q)$ the vector space of all harmonic homogeneous polynomials on $\mathbb{C}^{n}$ that have degree $p$ in $z$ and degree $q$ in $\bar{z}$. We let $\sigma$ be the rotation-invariant positive Borel measure on $S$ for which $\sigma(S)=1$. The space $L^{2}(S, \sigma)$ is the direct sum of the pairwise orthogonal spaces $H(p, q)$. Let $\pi_{p q}$ be the orthogonal projection of $L^{2}(S, \sigma)$ onto $H(p, q)$. Fix $p$ and $q$. To every $z \in S$ corresponds a unique $K_{z}$ in $H(p, q)$ that satisfies

$$
\left(\pi_{p q} f\right)(z)=\int_{s} f \bar{K}_{z} d \sigma \quad\left(f \in L^{2}(S, \sigma)\right) .
$$

We can define $\pi_{p q} \mu$ when $\mu \in \mathscr{M}(S)$. Consider now

$$
\operatorname{spec} \mu=\left\{(p, q) \in \mathbb{N} \times \mathbb{N}, \pi_{p q} \mu \neq 0\right\} .
$$

Let $\tau_{p q}$ be the restriction of the left regular representation of $U(n)$ on $L^{2}(S, \sigma)$ to $H(p, q)$, that is

$$
\left(\tau_{p q}(U) f\right)(l)=f\left(U^{-1} l\right) ; f \in H(p, q), U \in U(n), l \in S .
$$

The $\tau_{p q}$ are pairwise non-equivalent and they represent all irreducible representations of $U(n)$ which occur in $L^{2}(S, \sigma)$, and $n\left(\tau_{p q}\right)=q-p$, where $n\left(\tau_{p q}\right)$ is defined by the relation (5).

From Theorem 7.3 we then get,

COROLlary 7.8. Let $\Delta \subset \mathbb{N} \times \mathbb{N}$ be such that for all $N \in \mathbb{Z}$

$$
\{(p, q) \in \Delta, q-p \leq N\} \text { is a } \Lambda(1) \text {-set. }
$$

Then $\Delta$ is a Shapiro set.

We can improve Theorem 1.1 of [3]:

Corollary 7.9. Let $\Delta \subset \mathbb{N} \times \mathbb{N}$ be such that

(1) For each $N \in \mathbb{Z},\{(p, q) \in \Delta, q-p=N\}$ is finite,

(2) $\{q-p,(p, q) \in \Delta\}$ is bounded from below (or above).

Then $\Delta$ is a Shapiro set.

Let $K$ be a compact group whose center contains a copy of the unit circle. Let $H$ be a closed subgroup of $K$. We will extend Corollary 7.8 to the homogeneous space $K / H$. Functions (resp. measures) on $K / H$ can be identified with functions (resp. measures ) on $K$ which are right $H$-invariant. Let $\sigma$ be the $K$-invariant measure on $K / H$ for which $\sigma(K / H)=1$. If $\mu \in \mathscr{M}(K / H)$ 
is a right $H$-invariant measure on $K$ then $\pi_{\tau} \mu=d_{\tau} \chi_{\tau} \star \mu \quad(\tau \in \Sigma$ the dual of $K)$ is again $H$-invariant. The map $\pi_{\tau}: f \rightarrow d_{\tau} \chi_{\tau} \star f(\tau \in \Sigma)$ is an orthogonal projection of $L^{2}(K / H, \sigma)$ which is different from 0 if and only if $\tau$ occurs in the left regular representation of $K$ on $L^{2}(K / H, \sigma)$. For $f \in L^{1}(K / H, \sigma)$, consider

$$
\operatorname{spec} f=\left\{\tau \in \Sigma, \pi_{\tau} f \neq 0\right\} \text {. }
$$

The " homogeneous version" of Theorem 7.3 (see also Theorem 3.7 [3]) is

Corollary 7.10. Let $\Delta \subset \Sigma$ be such that for all $N \in \mathbb{Z}$,

$$
\{\tau \in \Delta, n(\tau) \leq N\} \text { is a } \Lambda(1) \text {-set . }
$$

Then for every $\Gamma \subset \Delta, B\left(L_{\Gamma}^{1}(K / H), \sigma\right)$ is $L^{p}$-closed $(0<p<1)$.

Note that for $K=U(n), H=U(n-1)$ then $K / H=S_{2 n-1}$.

Following [3] we can apply these results to bounded symmetric domains. Let $\Omega \in \mathbb{C}^{n}$ be a bounded symmetric domain, we may assume $\Omega$ to be convex and circular. Let $K$ be the stabilizer of 0 in the component of the identity of the group of holomorphic automorphisms of $\Omega$. The center of $K$ contains a copy of the circle. We can apply Corollary 7.10 to the BergmanShilov boundary $S$ of $\Omega$. Let $H^{2}(S)$ be the closure in $L^{2}(S, \sigma)$ of the holomorphic polynomials restricted to $S, H^{2}(S)$ is $K$-invariant under the left regular representation of $K$ in $L^{2}(S, \sigma)$. Let $\widehat{K}_{H o l}$ be the set of irreducible representations of $K$ which occur in $H^{2}(S)$. Let $H(p)$ be the space of holomorphic polynomials which are homogeneous of degree $p \quad(p \in \mathbb{N})$ restricted to $S . H(p)$ is $K$-invariant and decomposes as a finite sum of representations in $\widehat{K}_{h o l}$. If $\tau \in \widehat{K}_{H o l}$ then $n(\tau) \leq 0$ and $n(\tau)=-p$ if $\tau$ occurs in $H(p)$ (see (5) for the definition of $n(\tau)$ ). Therefore $\widehat{K}_{H o l}$ satisfies conditions (1) and (2) of Corollary 7.9 and $\widehat{K}_{H o l}$ is a Shapiro set.

\section{Acknowledgment}

I would like to thank M. Bozejko and S. Pichoridès for their helpful suggestions and G. Godefroy for many useful conversations.

\section{References}

[1] G. F. Bachelis and S. E. Ebenstein, 'On $\Lambda(p)$-sets', Pacific J. Math. 54 (1974), 35-38.

[2] J. Boclé, 'Sur la théorie ergodique', Ann. Inst. Fourier (Grenoble) 10 (1960), 1-45. 
[3] R. G. M. Brummelhuis, 'An F. and M. Riesz theorem for bounded symmetric domains', Ann. Inst. Fourier (Grenoble) 37 (1987), 139-150.

[4] __ ' 'Variations on a theme of Frederic and Marcel Riesz', Thesis.

[5] ___, 'A note on Riesz sets and lacunary sets', J. Austral. Math. Soc. (Series A) 48 (1990), 57-65.

[6] J. Diestel, Geometry of Banach spaces. Selected topics, Lecture Notes in Math. $\mathbf{4 8 5}$ (Springer, Berlin, 1975).

[7] R. E. Dressler and L. Pigno, 'Une remarque sur les ensembles de Rosenthal et Riesz', Note aux C.R.A.S. 280 (1975), 1281-1282.

[8] C. F. Dunkl and D. E. Ramirez, 'A family of countable compact $P_{\star}$ - hypergroups', Trans. Amer. Math. Soc. 202 (1975), 338-356.

[9] J. J. F. Fournier and K. A. Ross, 'Random Fourier series on compact abelian hypergroups', J. Austral. Math. Soc. (Series A) 37 (1984), 45-81.

[10] G. Godefroy, 'On Riesz subsets of abelian discrete groups', Israel J. Math. 61 (1988), 301-331.

[11] ___, 'On coanalytic families of sets in harmonic analysis', Illinois J. Math. 35 (1991), 241-249.

[12] P. Halmos, Measure theory (Van Nostrand, Princeton, 1950).

[13] V. P. Havin, 'Weak completeness of the space $L^{1} / H_{0}^{1}$ ', Vestnik Leningard Univ. 13 (1973), 73-81 (in Russian).

[14] E. Hewitt and K. A. Ross, Abstract Harmonic Analysis II (Springer, Berlin, 1970).

[15] R. I. Jewett, 'Spaces with an abstract convolution of measures', Adv. Math. 18 (1975), 1-101.

[16] F. Lust-Piquard, 'Ensembles de Rosenthal et ensembles de Riesz', C.R. Acad. Sci. Paris 282 (1976), 833-835.

[17] Y. Meyer, 'Spectres des mesures et mesures absolument continues', Studia Math. 30 (1968), 87-99.

[18] M. C. Mooney, 'A theorem on bounded analytic functions', Pacific J. Math. 43 (1972), 457-463.

[19] J. F. Price, 'Non a sono inoemi infiniti di tipo $\Lambda(p)$ per $S U(2)$ ', Boll. Un. Mat. Ital. 4(4) (1971), 879-881.

[20] D. Rider, ' $S U(n)$ has no infinite local $\Lambda_{p}$ sets', Boll. Un. Mat. Ital. 12(4) (1975), 155-160.

[21] W. Rudin, Real and complex analysis (Mc Graw-Hill, New York, 1966).

[22] J. Shapiro, 'Subspaces of $L^{p}(G)$ spanned by characters, $0<p<1$ ', Israel J. Math. 29 (1978), 248-264.

[23] R. Spector, 'Aperçu de la théorie des hypergroupes', in: Analyse harmonique sur les groupes de Lie. sém. Nancy-Strasbourg 1973-1975, Lecture Notes in Math. 497 (Springer, Berlin, 1976) pp. 643-673.

[24] R. C. Vrem, 'Harmonic analysis on compact hypergroups', Pacific J. Math. 85 (1979), 239-251.

\section{Université de Mons}

Faculté des Sciences

Avenue Maistriau 15

B 7000 Mons

Belgique 